Available online at GSC Online Press Directory

GSC Biological and Pharmaceutical Sciences

e-ISSN: 2581-3250, CODEN (USA): GBPSC2

Journal homepage: https://www.gsconlinepress.com/journals/gscbps

(RESEARCH ARTICLE)

\title{
Measurement of the peritumoral edema and tumor volume ratio in differentiating malignant primary and metastatic brain tumor
}

\author{
Aulia Hanum ${ }^{1, *}$, Achmad Bayhaqi Nasir Aslam ${ }^{1}$, Yuyun Yueniwati ${ }^{1}$, Diah Prabawati Retnani ${ }^{2}$ and Nanik \\ Setjowati ${ }^{3}$ \\ ${ }^{1}$ Radiology Department, Faculty of Medicine, Universitas Brawijaya, Malang, Indonesia. \\ 2 Pathology Anatomy Department, Faculty of Medicine, Universitas Brawijaya, Malang, Indonesia. \\ 3 Public Health Department, Faculty of Medicine, Universitas Brawijaya, Malang, Indonesia.
}

Publication history: Received on 12 September 2020; revised on 31 October 2020; accepted on 03 November 2020

Article DOI: https://doi.org/10.30574/gscbps.2020.13.2.0295

\begin{abstract}
Malignant primary and metastatic brain tumors are group of malignancies radiologically difficult to distinguish between one another. Meanwhile, the treatment regimens between the two entities are very different. The right regimen can maintain patient's survival. MRI is the modality of choice for diagnosing brain tumors; although, malignant primary brain tumors and solitary metastases appear similar on conventional MRI. The difference in the pathophysiology of peritumoral edema in malignant primary and metastatic brain tumors has the potential for differentiation of the two entities. In malignant primary brain tumors, tumor cell infiltration occurs in the edema area, meaning that the peritumoral edema is narrower than that of the metastases. This study analyzed the ratio of peritumoral edema volume to tumor (EP/T volume ratio) in malignant primary and metastatic brain tumors by means of MRI examination with a cross-sectional design, using MRI data on FLAIR and T1WI sequences with contrast in malignant brain tumor of patients that have been pathologically proven. Then, volume contouring was performed on peritumoral edema (EP) and tumor $(\mathrm{T})$, and comparation was done to obtain the EP/T volume ratio. The ratio of EP/T volume data in both groups was analyzed using the Mann-Whitney test with the SPSS 22 software. The results of statistical analysis revealed that the $\mathrm{EP} / \mathrm{T}$ volume ratio of the malignant primary brain tumor group was smaller with a median value (max-min) of 1.1 (5.650.17) and in the metastatic group, 2.3 (64.03-0.09). There was a significant difference in the EP/T volume ratio between the two groups, which the brain metastatic tumor group have a double ratio of EP/T with a value of $p=0.008(p<0.05)$.
\end{abstract}

Keywords: MRI of brain tumor; Ratio of peritumoral edema and tumor; Malignant primary brain tumor and metastases

\section{Introduction}

Brain tumors are a group of malignancies that can generally be classified into primary and secondary brain tumors. Primary brain tumors grow from cells normally in the brain. Secondary brain tumors are tumors originating from the spread or metastasis of malignancy in other organs [1]. The optimal treatment regimens for primary brain tumors and metastatic brain tumors are very different; therefore, differentiating the two entities is very important [2]. Brain magnetic resonance imaging (MRI) examination with contrast is the neuroimaging gold standard in suspected brain tumors [3]. However, in some cases, especially when the lesion is solitary and no other malignancy is found, conventional MRI examination alone is difficult when it comes to distinguishing primary from metastatic brain tumors [4].

\footnotetext{
${ }^{*}$ Corresponding author: Aulia Hanum

Department of Radiology, Faculty of Medicine, Universitas Brawijaya, Malang, Indonesia.

Copyright (C) 2020 Author(s) retain the copyright of this article. This article is published under the terms of the Creative Commons Attribution Liscense 4.0.
} 
Peritumoral edema can be an important indicator of tumor malignancy when radiologists are evaluating radiological images. Peritumoral edema in malignant brain tumors is mainly due to vasogenic and cytotoxic factors. The pathophysiological differences between malignant primary and metastatic brain tumors allow for a significant difference in the ratio of peritumoral edema volume to tumor (EP/T volume ratio) that is present in malignant primary and metastatic brain tumors. The volume ratio can be used as a valuable marker for imaging brain tumors using conventional MRI sequences, such as post-contrast T1WI and FLAIR [5].

The pathophysiological differences in the formation of peritumoral edema between malignant primary brain tumors and metastases underlie the authors' intention to write this paper. This study was conducted by analyzing the images produced by conventional MRI imaging through assessing the difference in the EP/T volume ratio of malignant primary and metastatic brain tumors.

\section{Research methods}

\subsection{Sample population}

The study population comprised patients with malignant primary and metastatic brain tumors who had their MRI examination results and pathology examination results of brain tissue or other organs indicating malignancy.

The inclusion criteria of this study were malignant brain tumor patients who had their MRI results and had their pathology examined (without immune histo chemical examination). Meanwhile, the exclusion criteria were patients with brain tumors who had their MRI examination results but not their pathological results; patients with malignant primary brain tumors with extra axial location or outside of the brain parenchyma; and patients with secondary brain tumors that were direct invasion from their primary tumor.

\subsection{Research methods}

This cross-sectional retrospective observational study has been reviewed and approved by the ethics committee of dr. Saiful Anwar Malang Hospital No. 400/069 / K.3 / 302/2020. The study was conducted from March to June 2020. Data were taken retrospectively from the medical records of patients with malignant brain tumors who had MRI examinations and pathology examinations.

We made use retrospective data from the MRI examination at the radiology installation of dr. Saiful Anwar Malang Hospital and Persada Hospital in Malang which were by non-researchers. In this data, the measurement of the peritumoral edema volume in the T2 FLAIR sequence and the tumor volume in the T1WI with contrast sequence was carried out using the IntelliSpace Portal Philips software on the CT viewer menu in three dimensions (3D).

\subsection{Statistics analysis}

All data obtained from the research results were stored in computer files. The comparison of the EP/T volume ratio between malignant primary and metastatic brain tumors using MRI examination was analyzed using non-parametric test (Mann-Whitney test) due to the abnormal distribution of the data. Statistical analysis in this study employed the SPSS 22 computer software.

\subsection{Research result}

This study spanned from March to June of 2020 with a total sample size of 61 meeting the research criteria.

Table 1 Sample Characteristics based on Gender and Age.

\begin{tabular}{|l|l|l|l|}
\hline Demographic Characteristics & $\begin{array}{l}\text { Malignant primary } \\
\text { brain tumor (n= 11) }\end{array}$ & $\begin{array}{l}\text { Metastatic brain tumor } \\
(\mathbf{n = 5 0 )}\end{array}$ & p-value \\
\hline 1. Age (years) mean \pm SD & $51.18 \pm 19.31$ & $45.54 \pm 8.97$ & \multirow{2}{*}{0.777} \\
\hline 2. Gender & $4(6.6 \%)$ & $8(13.1 \%)$ & \\
\hline Male & $7(11.5 \%)$ & $42(68.9 \%)$ & \\
\hline Female & \multicolumn{2}{|l|}{0.124} \\
\hline
\end{tabular}


The study sample consisted of 11 samples of malignant primary brain tumors and 50 samples of metastatic brain tumors. Most of the research subjects were female, accounting for $80.3 \%$ with the remaining $19.7 \%$ were male (table 1).

The age range of patients in the group of patients with malignant primary brain tumors was between the ages of 35-80 years, mostly comprising 44-year-olds. Meanwhile, the age range in the metastatic group was 35-70 years with many of which were 50-year-olds.

Table 2 Sample Characteristics Based on Tumor Origin.

\begin{tabular}{|c|l|l|}
\hline Characteristic & Frequency & Percentage (\%) \\
\hline Malignant primary brain tumor & 11 & 18.0 \\
\hline $\begin{array}{l}\text { Metastatic Tumors, with primary } \\
\text { tumor origin }\end{array}$ & 50 & 82.0 \\
\hline \multirow{2}{*}{$\begin{array}{l}\text { Lung cancer } \\
\text { Breast cancer } \\
\text { Lymphoma } \\
\text { Cervix cancer } \\
\text { Ovarium cancer }\end{array}$} & 23 & 37.7 \\
\cline { 2 - 3 } Unknown & 21 & 34.5 \\
\cline { 2 - 3 } & 1 & 1.6 \\
\cline { 2 - 3 } & 1 & 1.6 \\
\cline { 2 - 3 } & 2 & 3.3 \\
\cline { 2 - 3 } & 2 & 3.3 \\
\hline
\end{tabular}

Patients with malignant primary brain tumors in this study had biopsy from their brain tissue with results showing 7 samples of glioblastoma (WHO grade IV), 3 samples of anaplastic astrocytoma (WHO grade III), and 1 anaplastic oligodendroglioma (WHO grade III). Patients with metastatic brain tumors in this study were confirmed by brain tissue biopsy (1 sample), CSF fluid biopsy (9 samples), and the remaining 40 samples were patients with multiple intraparenchymal brain lesions and had primary tumors in other organs that were confirmed by pathological examination. In the metastatic group, the various origins of tumor were identified, i.e. lung, breast, ovary, cervix, lymphoma, and 2 samples had unidentifiable primary tumor from which it originated (Table 2).

The location of the 11 lesions of the malignant primary brain tumor group is on supratentorial region of the brain (100\%); whereas, 41 (67.2\%) lesions of the metastatic brain tumor group were on the supratentorial region, 8 (13.1\%) lesions were on infratentorial region, and 1 lesion (1.6\%) was on the brainstem (figure 1).

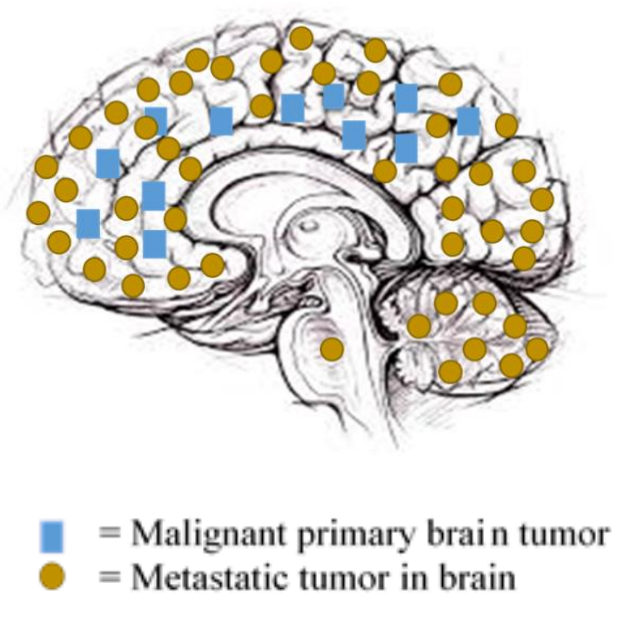

Figure 1 Distribution of Tumor Locations 
The Kruskal-Wallis statistical test was carried out to determine whether the the location of the lesion effect the peritumoral edema, because the tumoral edema data were not normally distributed, and a significance value of 0.673 was obtained ( $p>0.05)$. This means, statistically, the location of the lesion in the brain has no effect on the volume of peritumoral edema.

The proportion of tumor cystic lesions in the malignant primary and metastatic brain tumor groups has different percentages (Figure 2).

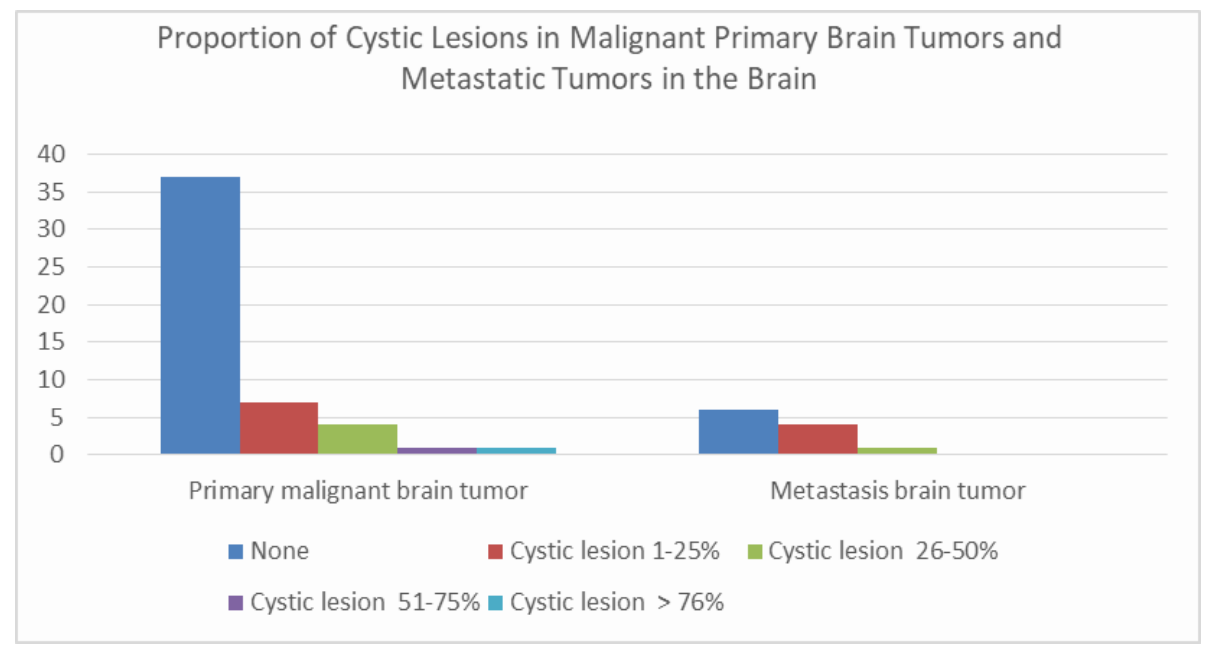

Figure 2 Proportion of Cystic Lesions in Malignant Primary and Metastatic Brain Tumors.

Kruskal-Wallis statistical test was performed and the significance value was 0.119 ( $p>0.05)$, which means that, statistically, the proportion of cystic lesions in the tumor did not affect the volume of peritumoral edema.

Contouring of FLAIR and T1WI MRI images with contrast sequences was performed on 61 patient samples from both groups. In the FLAIR sequence, the contouring area that looks hyperintense is the tumor area and its peritumoral edema; in the T1WI with contrast sequence contouring, the hyperintense area represent the tumor area.
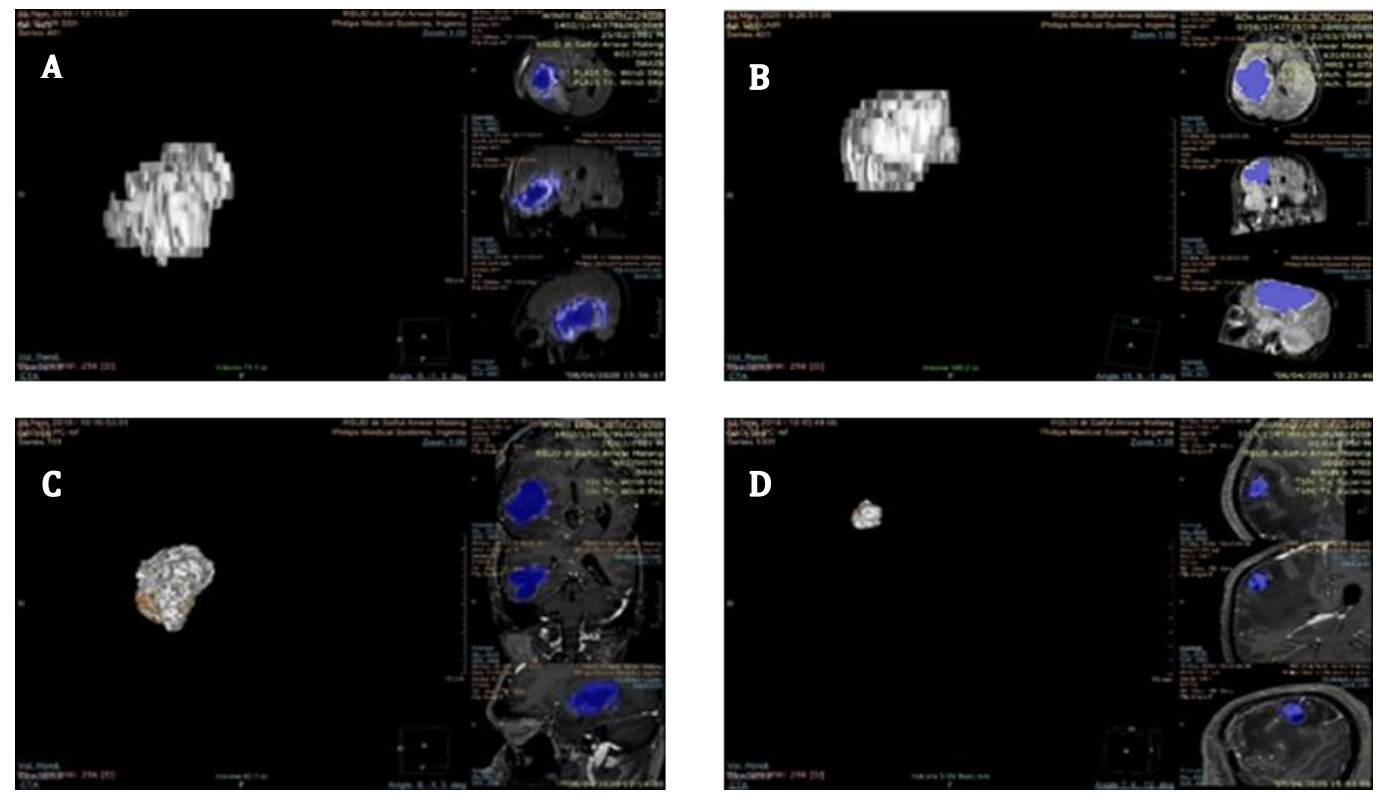

Figure 3 Contouring on MRI

Contouring of the FLAIR sequence in patients with malignant primary brain tumors (A) and in metastatic brain tumors (B). Contouring of T1WI sequences in patients with malignant primary brain tumors (C) and in metastatic brain tumors (D). 
Based on the data obtained, it is known that the EP/T volume ratio data in the group of patients with malignant primary brain tumors has a smaller value with the median (max-N. min) of 1.1 (5.65-0.17) compared to the metastatic group that has a median value (max-min) of 2.3 (64.03-0.09). There was a significant difference in the EP/T volume ratio between the two groups, which the brain metastatic tumor group have a double ratio of EP/T with a value of $\mathrm{p}=0.008$ $(\mathrm{p}<0.05)$.

Table 3 Data Analysis

\begin{tabular}{|c|c|c|c|c|c|}
\hline \multirow[b]{2}{*}{ Variable } & \multicolumn{2}{|l|}{ Mean \pm SD } & \multicolumn{2}{|c|}{ Median (Max - Min) } & \multirow[b]{2}{*}{$\mathbf{p}$} \\
\hline & $\begin{array}{l}\text { Malignant } \\
\text { primary } \\
\text { brain tumor }\end{array}$ & $\begin{array}{l}\text { Metastatic } \\
\text { brain tumor }\end{array}$ & $\begin{array}{l}\text { Malignant } \\
\text { primary } \\
\text { brain tumor }\end{array}$ & $\begin{array}{l}\text { Metastatic } \\
\text { brain tumor }\end{array}$ & \\
\hline $\begin{array}{l}\text { Volume of } \\
\text { peritumoral } \\
\text { edema (cc) }\end{array}$ & $86.69 \pm 73.25$ & $40.24 \pm 62.64$ & $\begin{array}{l}108.8 \\
(196.7-4.76)\end{array}$ & $\begin{array}{l}7.60 \\
(208.4-0.19)\end{array}$ & 0.012 \\
\hline $\begin{array}{l}\text { Volume of } \\
\text { tumor }(\mathrm{cc})\end{array}$ & $73.53 \pm 43.71$ & $11.51 \pm 18.82$ & $\begin{array}{l}93.1 \\
(132.7-3.44)\end{array}$ & $\begin{array}{l}3.09 \\
(81.2-0.17)\end{array}$ & 0.000 \\
\hline $\begin{array}{l}\mathrm{EP} / \mathrm{T} \text { volume } \\
\text { ratio }\end{array}$ & $1.46 \pm 1.54$ & $5.42 \pm 10.29$ & $\begin{array}{l}1.1 \\
(5.65-0.17)\end{array}$ & $\begin{array}{l}2.32 \\
(64.03-0.09)\end{array}$ & 0.008 \\
\hline
\end{tabular}

Peritumoral edema volume data for the malignant primary brain tumor group shows higher median value (max- min) of 108.8 (196.7-4.76), while in the metastatic group, it is $7.6(208.4-0.19)$ cc with $p$ value $=0.012(p<0.05)$. Moreover, the median value (max-min) of tumor volume for the malignant primary brain tumor group was relatively much greater with figure showing 93.1 (132.7-3.44) cc compared to the tumor volume for the metastatic group displaying median value (max-min) equal to $3.09(81.2-0.17)$ cc with $\mathrm{p}$ value $=0.000(\mathrm{p}<0.05)$.

To see the suitability between the diagnosis made through MRI examination and the pathology as gold-standard diagnosis in this study, we made a $2 \times 2$ suitability table, which was then tested for statistical differences. Through the McNemar's test, a significance value of $0.035(\mathrm{p}<0.05)$ was obtained, indicating that there is a significant difference between diagnosis through MRI examination and diagnosis by pathology.

Table 4 Cross-tabulation of MRI diagnosis with final diagnosis.

\begin{tabular}{|l|l|l|l|}
\cline { 3 - 4 } \multicolumn{2}{c|}{} & Final Diagnosis \\
\cline { 3 - 4 } $\begin{array}{l}\text { MRI } \\
\text { Diagnosis }\end{array}$ & Primary malignant brain tumor & Metastasis brain tumor \\
\cline { 2 - 4 } & Primary malignant brain tumor & 8 & 12 \\
\hline
\end{tabular}

\section{Discussion}

In malignant brain tumors, both primary and metastatic are always followed by severe peritumoral edema. However, measurement of the ratio of peritumoral edema volume to the tumor has not been widely used for distinguishing the two types of malignant tumors [5].

Through conventional sequence MRI examination, the features of malignant primary and metastatic brain tumors can be very similar. In distinguishing the two entities, it is often difficult, even with the combination of several advance sequences such as MR spectroscopy, diffusion, and perfusion [6]. In this study, there was a significant difference in the $\mathrm{EP} / \mathrm{T}$ volume ratio between the two groups. Based on the theory, in malignant primary brain tumors, peritumoral edema occurs in the form of vasogenic edema accompanied by infiltration of tumor cells in the edema area. In addition, malignant primary brain tumors also involve cytotoxic edema, which is the effect of glial changes in brain tissue and causes astrocytic swelling [7]. The rapid mass growth in malignant primary brain tumors requires angiogenesis to support its growth, resulting in increased activity of vascular endothelial growth factor (VEGF). An increase in VEGF 
activity causes disruption of the blood brain barrier and causes peritumoral vasogenic edema. However, due to the presence of hypercellularity in the intracellular space and cytotoxic edema, the extracellular fluid levels are reduced, causing vasogenic edema that is less extensive than the size of the lesion in malignant primary brain tumors [8]. In contrast, metastatic brain tumors multiply to form localized masses in the brain [9]. The occurrence of metastatic brain tumors always has high VEGF levels which causes extensive vasogenic edema because metastatic brain tumors does not involve tumor cell infiltration in the edema area [10].

The statistical results in this study show that in malignant primary brain tumors, the EP/T volume ratio is smaller than the metastatic group. In primary brain tumors, the median value (max-min) of EP/T volume ratio is 1.1 (5.65-0.17) and in the metastatic group, it is $2.32(64.03-0.09)$ with $p$ value $=0.008(\mathrm{p}<0.05)$. This is in accordance with the theory and hypothesis stating that metastatic tumors in the brain have a wider area of edema and are often disproportionate to the size of the tumor [5]. This research is expected to increase the diagnostic value for the imaging of malignant brain tumors through conventional sequence MRI, even though more-extensive research should be done to find the cut-off ratio between the two groups.

The components that constitute the EP/T volume ratio in this study are peritumoral edema volume and tumor volume. In this study, it was found that the volume of peritumoral edema in the malignant primary brain tumor group was greater than the volume of peritumoral edema in the metastatic group, which are consecutively 108.8 (196.7-4.76) cc and 7.60 (208.4-0.19) cc with p value $=0.012(\mathrm{p}<0.05)$. Also, the median value (max-min) of tumor volume in the malignant primary brain tumor group was relatively much greater with a value showing 93.1 (132.7-3.44) cc compared to the median value of tumor volume in the metastatic group of $3.09(81.2-0.17)$ cc with $p$ value $=0.000(p<0.05)$.

Factors possibly influencing the formation of peritumoral edema volume are the location of the tumor and the proportion of cystic lesions in the tumor. In a study by Zhou et al in 2016, it was found that peritumoral edema was smaller in the cerebellum and in lesions with a large cystic component. However, in this study, there was no correlation of the location of the lesion and the proportion of cystic lesions to the volume of peritumoral edema [5]. This difference may have been due to the diversity of the different sizes and the unequal distribution of lesion locations among the study samples.

This study also assessed the suitability between the radiological diagnosis made from the MRI examination and the final diagnosis by pathology as the gold standard. Through the McNemar's test, a significance value of $0.035(\mathrm{p}<0.05)$ was obtained, indicating that there is a significant difference between diagnosis through MRI examination and final diagnosis.

According to the suitability test, 46 samples (75\%) had the same diagnosis, be it through MRI examination or the final diagnosis. Meanwhile, 15 samples (25\%) from 4 patients had an MRI diagnosis that was different from the final diagnosis. A total of 12 samples (which came from 1 patient) were diagnosed with brain infection on the MRI examination. However, it turned out that the patient's pathology revealed metastatic tumors in the brain. In addition, as many as 3 samples (which came from 3 patients) were diagnosed with metastatic brain tumors from the MRI examination, while in reality, the patients actually suffered from malignant primary brain tumors. Since the therapeutic protocols are very different in these two entities [2,11), further research for adding diagnostic value to MRI examinations is really crucial.

\subsection{Research Limitations}

This study employed retrospective data, so it was difficult to make the scanning protocol uniform, especially in the FLAIR and T1WI with contrast sequences. In addition, in this study, there are no data regarding the history of medications consumed by the patients which might affect the formation of peritumoral edema.

\section{Conclusion}

This study found that the malignant primary brain tumor group has a greater tumor volume and a significantly smaller peritumoral edema volume than the metastatic tumor group. Therefore, the volume ratio of peritumoral edema to tumor in the malignant primary brain tumors is significantly smaller than that of metastatic tumors.

The measurement for the volume of both tumor and peritumoral edema in this study was carried out in three dimensions by means of the Philips IntelliSpace Portal software on the CT viewer menu. 


\section{Compliance with ethical standards}

\section{Acknowledgments}

Thankful to the Radiology Department and Pathology Department, Faculty of Medicine, Universitas Brawijaya - Saiful Anwar Hospital Malang for the opportunity given to make a research

Many thanks to Prof Dr Hamzaini Abdul Hamid, MBBch, DrRad for suggestion and correcting the manuscript.

\section{Disclosure of conflict of interest}

This research doesn't have a conflict of interest.

\section{Statement of informed consent}

This research has been accepted by the patient and the patient has been signed an informed consent form for the anonymous publication of medical data.

\section{References}

[1] Perkins,A. Liu, G. Primary Brain Tumors in Adults: Diagnosis and Treatment - American Family Physician. Am. Fam. Physician. 2016; 93, 211-218.

[2] Kamson, D. O. et al. Differentiation of glioblastomas from metastatic brain tumors by tryptophan uptake and kinetic analysis: A positron emission tomographic study with magnetic resonance imaging comparison. Mol. Imaging. 2013; 12, 327-337.

[3] Blystad, I. et al. Quantitative MRI for analysis of peritumoral edema in malignant gliomas. PLoS One. 2017; 12, 112.

[4] Fink, K. R. \& Fink, J. R. Imaging of brain metastases. Surg. Neurol. Int. 4, S209-19 (2013).

[5] Zhou, C. et al. Segmentation of peritumoral oedema offers a valuable radiological feature of cerebral metastasis. Br. J. Radiol. 2016; 89.

[6] Bauer AH, Erly W, Moser FG, Maya M, N. K. Differentiation of solitary brain metastasis from glioblastoma multiforme: a predictive multiparametric approach using combined MR diffusion and perfusion. Neuroradiology. 2015; 57, 697-703.

[7] Engelhorn, T. et al. Cellular characterization of the peritumoral edema zone in malignant brain tumors. Cancer Sci. 2009; 100, 1856-1862.

[8] Fabian, M. \& Walker, M. The neuropathology of brain metastases. Diagnostic Histopathol. 2019; 25, 1-7.

[9] Lemercier, P., Maya, S. P., Patrie, J. T., Flors, L. \& Leiva-Salinas, C. Gradient of apparent diffusion coefficient values in peritumoral edema helps in differentiation of glioblastoma from solitary metastatic lesions. Am. J. Roentgenol. 2014; 203, 163-169.

[10] Alonso, A. S. Advanced Neuroimaging in Brain Tumors : Diffusion, Spectroscopy, Perfusion and Permeability MR imaging for the evaluation of tumor characterization and surgical treatment planning . (2011).

[11] Magnetic Resonance Imaging (MRI) as an early detector of cognitive impairment. J Neurosci Rural Pract. 2018;9(3):350-3. 